\title{
El Concepto de Participación de los \\ Padres en el Proceso de Enseñanza Aprendizaje: La Perspectiva de Agentes Comprometidos
}

\section{Conceptualizations About the Parent Involvement on the Teaching-Learning Process Actors}

\author{
Gracia Navarro Saldaña * \\ Pamela Vaccari Jiménez \\ Tatiana Canales Opazo
}

\section{Resumen}

Para investigar el concepto de participación de los padres y /o apoderados en el proceso de enseñanza - aprendizaje de los hijos, se consultó la opinión a padres, profesores y autoridades educativas, utilizando metodologías cualitativas, con personas pertenecientes a escuelas urbanas y rurales de la Región del Bío Bío.

Hay diferencias de conceptualización entre los directivos y otras personas de la muestra, ya que los primeros analizan el tema desde una posición más teórica que los que viven realmente la relación entre escuela y familia. Otros resultados indicaron que la participación de los padres sería vista como una amenaza por los profesores y que, para los padres, ésta aún se restringe a aspectos como la colaboración instructiva desde su hogar.

Palabras claves: Reforma Educativa, Participación, Relación Familia - Escuela.

\section{Abstract}

To investigate the concept of parent involvement in their children's education, parents, teachers, and educational authorities were asked to give their views through qualitative methodologies. Participants belong to urban and country schools of the región del Bío Bío.

There are differences in the conceptualizations of the board members and the others participants of the study, because they have a point of view more theoretic than who lives the relationship between home school. Other results indicate than the parent involvement should be taken like a threaten by the teachers, and this is still restricted to the instructive aspect from the home to the parents.

Keywords: Educational Reform, Parent involvement, Home-school relationship.

* Departamento de Psicología, Universidad de Concepción. gnavarro@udec.cl 


\section{Introducción}

En las últimas décadas se ha ido realizando un significativo proceso de cambio en la estructura socioeducacional chilena. La generación de éstos cambios modernizadores han tomado cuerpo a través de la Reforma Educacional, impulsando un nuevo estilo de gestión escolar que busca dinamizar el proceso de enseñanza aprendizaje, mejorando la calidad y equidad de la educación.

En este marco, uno de los ejes centrales de modernización del estado que recae también en la reforma educativa es asignar importancia a la descentralización de las decisiones y por lo tanto dar paso a la participación efectiva. En el contexto de las políticas educativas se han implementado varias iniciativas como el PADEM (Plan Anual de Desarrollo de la Educación Municipal, Ley 19.410), la inserción de los OFT (Objetivos Fundamentales Transversales, en el marco de los PEI -proyectos educativos institucionales), la delegación de facultades (Ley 19.410) a los Directores y por consiguiente a la comunidad educativa, la Ley de Donaciones con Fines Educativos (Ley 19.247) y los Programas de Mejoramiento Educativo (PME), son algunos de estos ejemplos (MINEDUC, 1998).

La Participación es considerada como uno de los pilares de la Democracia y por eso la Reforma Educativa Chilena expresa que las escuelas deben ayudar a la formación de criterios democráticos y participativos de los alumnos, por lo tanto, se abre el espacio para la comunidad y las familias para que el acto de "educar" sea un proceso integral en el que se involucren todos estos estamentos (MINEDUC, 1998). De acuerdo a esto, existen muchos autores que han reflexionado en torno al tema de la participación de los padres y/o apoderados en el proceso de enseñanza - aprendizaje de los hijos, sobre sus beneficios y las dificultades de su puesta en marcha. Por ejemplo, Fernández y Guerrero (1996) opinan que la participación en la escuela es tomar parte activa en la elaboración y desarrollo de sus líneas de acción con todos los niveles que intervienen, gobierno, municipio y escuela. También el MINEDUC (1995) destaca que la escuela como referente de la comunidad, es más participativa si logra ser autónoma en sus aspectos administrativos, financieros y pedagógicos, apuntando a que la escuela sería más efectiva si convoca a la familia en sus procesos, además de incorporar a la comunidad y a sus docentes en constante formación profesional.

Para diversos autores el proceso educativo comienza en la familia por ser ésta el primer agente implicado en este proceso, y por lo mismo la influencia de la familia es muy relevante en los resultados instruccionales, formativos y afectivos logrados por parte del educando (Arancibia, 1998; Dubois, Eitel y Felner, 1994; Fernández y Salvador, 1994; Gento, 1994; González, 1996; Pulkinen, 1982; Rippia, 1994; Uribe, 1992; citados todos en Pardo et al., 1998).

Históricamente, la familia se encargaba de la transmisión de la memoria social de la comunidad, pero luego con el surgimiento de las instituciones educativas formales, este derecho y deber fue delegado a las últimas y conforme transcurrió el tiempo, la familia pasó a adoptar un rol de continuadora de la labor docente en el hogar, es decir, de colaboración (Cantón, 1996; Neira, 1994). Hoy en día se rescata el valor primordial que cumplen los padres y/o apoderados en el proceso de enseñanza aprendizaje de los hijos, como en su rendimiento, orientación hacia el logro, el autoconcepto académico y el éxito escolar. La participación de la familia tiene que ver también con hacer válidos los ideales democráticos, con aprovechar mejor los servicios educativos, superar la pobreza y elevar la igualdad de oportunidades, en definitiva, con la descentralización de las 
decisiones y con hacer una educación más pertinente a la realidad de la gente, en que se dé una articulación armónica entre familia y escuela. Los autores como Arancibia (1997) dicen que la escuela es la encargada de los procesos más colectivos, mientras que la familia prestaría una atención más individual, también se señala que la familia satisface las necesidades emocionales de sus hijos, mientras que la escuela satisface además las necesidades intelectuales, ya que el profesor no sólo debe aceptar a los niños y sus emociones, sino que también debe formar y fomentar su aprendizaje.

La Reforma Educativa Chilena, al proponer la participación de los padres y/o apoderados de familias y estudiantes, tiene como base el supuesto de que la familia es experta en la educación de sus hijos y que es por lo tanto, un recurso pedagógico educativo, al manejar la información que puede ser de beneficio para nutrir y complementar la práctica pedagógica del profesorado y que además es una gran red de apoyo para los maestros, con la cual se puede planificar como ejecutar acciones que ayuden a los estudiantes (Revista de Educación, 1998).

Sin embargo al parecer el discurso sobre la necesidad de incorporar a los padres al proceso de enseñanza - aprendizaje, no ha tenido contenido significativo ni para los padres y/o apoderados, ni para los propios docentes, ya que en la práctica aún son escasas las experiencias de procesos que se ejecuten con la participación de éstos (Assaél, Cerda y Rodas, 1996).

Aún queda mucho por hacer para que la participación sea una realidad en las escuelas: se deben crear otros códigos para la interrelación familia - educación, generando espacios de encuentro más atractivos para los padres (Fernández y Guerrero, 1996; Pardo et al.,1998). Es necesario que los profesores conozcan y comprendan más el entorno social de sus alumnos $\mathrm{y}$, como lo sostienen MINEDUC (1995) y Pardo et al. (1998), las familias deberían aportar sus ideas y hacerse responsables de la educación de sus hijos en el sistema.

Conforme a lo anterior y dado que el planteamiento de la reforma no explicita las acciones concretas de participación, se formula el siguiente problema de investigación: ¿Cómo conceptualizan las autoridades educativas, los profesores y los mismos apoderados la participación de los padres en el proceso de enseñanza - aprendizaje de los hijos y en qué medida difiere la definición que de este concepto tienen los distintos actores, atendiendo a la procedencia geográfica (urbano - rural) y nivel jerárquico de éstos?

\section{Método}

\section{a) Sujetos}

Se seleccionó un grupo de padres, uno de profesores y uno de autoridades educacionales, pertenecientes a 2 escuelas urbanas y 2 rurales, de las comunas de Chiguayante y Coelemu, respectivamente. Los establecimientos fueron sorteados de todas las escuelas urbanas de la comuna de Chiguayante y de todas las escuelas rurales de la comuna de Coelemu. Los tres grupos estuvieron formados como sigue:

Padres: Formado por 36 sujetos, apoderados de kinder, cuarto básico y octavo básico. La mitad perteneciente a escuelas del sector urbano y los otros 18 del sector rural. De éstos, había 9 padres clasificados como participativos (que pertenecen a la directiva de microcentro: presidente, secretario y tesorero, con el supuesto de base que padre participativo es aquél que asume alguna responsabilidad concreta dentro del establecimiento educativo) y 9 clasificados como no 
participativos (que no pertenecen a la directiva de microcentro) en cada sector.

Profesores: Formado por 56 docentes. 15 pertenecientes a las escuelas rurales de Coelemu y 41 de las escuelas urbanas de Chiguayante.

Autoridades educativas: Formado por 10 personas. Dos Jefes de DAEM (uno de cada comuna); 4 supervisores de los Departamentos Provinciales de Educación (uno para cada escuela) y 4 Directores de Establecimientos educacionales (uno de cada escuela).

\section{b) Instrumentos}

Considerando el criterio de obtener el máximo de información posible acerca del concepto que se está manejando en la comunidad; la cantidad de personas involucradas; su disponibilidad de tiempo para participar en la investigación y los recursos disponibles para realizarla , se utilizó los siguientes instrumentos:

Cuestionario para profesores, de respuesta directa post codificado, elaborado para la investigación, formado por una sola pregunta abierta: “ ¿Cómo cree usted que debiera ser la relación entre la familia y la escuela o entre la escuela y la familia?". De aplicación colectiva, en un lapso de 15 minutos, aproximadamente.

Entrevista individual, semiestructurada, para autoridades educacionales, elaborada a partir de la pregunta base: ¿Cómo cree usted que debería ser la relación entre la familia y la escuela o entre la escuela y la familia?

\section{c) Procedimiento}

- Padres: El procedimiento utilizado fue el grupo de discusión, en base a la siguiente pregunta: ¿Cómo cree usted que debería ser la relación entre la fa- milia y la escuela o entre la escuela y la familia?

Se trabajó con cuatro grupos constituidos de la siguiente manera:

Grupo 1: 9 padres participativos (3 de cada curso considerado), sorteados de las escuelas rurales de Coelemu.

Grupo 2: 9 padres no participativos (3 de cada curso considerado), sorteados de las escuelas rurales de Coelemu.

Grupo 3: 9 padres participativos (3 de cada curso considerado), sorteados de las escuelas urbanas de Chiguayante.

Grupo 4: 9 padres no participativos (3 de cada curso considerado), sorteados de las escuelas urbanas de Chiguayante.

Los padres fueron invitados a participar a través de una tarjeta entregada al profesor, por intermedio del director del establecimiento. Ambos grupos se efectuaron en las aulas de las escuelas participantes, recopilando inicialmente la discusión mediante grabadora, previa autorización de los participantes.

Profesores: A los profesores de las escuelas rurales y urbanas se les pidió que respondieran el cuestionario en reunión de Consejo de profesores, en un lapso aproximado de 15 minutos.

Autoridades educacionales: A cada autoridad se realizó la entrevista semiestructurada en el tiempo y lugar que el entrevistado estimó conveniente. Cada entrevista tuvo una duración aproximada de una hora; fue grabada (previa autorización del entrevistado) y posteriormente transcrita al papel.

Para no inducir a los participantes en cuanto al concepto que manejan de participación de los padres en el proceso de enseñanza aprendizaje de los hijos, no se preguntó directamente por este concepto, sino so- 
bre su percepción de la relación familia - escuela o escuela familia. Se preguntó por estas dos relaciones para no inducir una direccionalidad en ella.

Se utilizó el procedimiento de análisis de contenido. Luego de leer cada una de las respuestas obtenidas, se formó categorías y subcategorías de análisis que agruparan las respuestas según las temáticas presentadas por los grupos de estudio. Luego se tabuló y calculó para cada grupo el porcentaje de opiniones correspondientes a cada categoría y subcategoríasCada subcategoría con su porcentaje de respuestas emitidas se expone acompañado por una opinión representativa.

\section{Resultados}

A partir del análisis de contenidos se encontró las ocho categorías de respuesta que se expone a continuación, acompañadas de opiniones representativas:

\section{Categorías encontradas}

a) Relación familia - escuela de acuerdo a su naturaleza

- Relación familia - escuela entendida como un deber: cuando el tomar parte en las actividades escolares es percibido como obligatorio. "Nosotras como mamás tenemos la obligación de darles educación a nuestros hijos" ; "Creo que hay una responsabilidad social que tampoco podemos eludir, en democracia es una obligación participar y es la obligación de uno asumir cuáles son sus derechos y deberes"

- Relación familia - escuela entendida como un compromiso: cuando el tomar parte en las actividades escolares es percibido como una acción realizada por propia motivación. "Cuando los padres participan en la formulación de proyectos educativos, sienten que éstos les pertenecen y se comprometen más con la escuela"; "Debe tratarse de un proceso de identificación con la escuela"

b) Relación familia - escuela desde el punto de vista de quien la inicia.

- Relación familia - escuela iniciada por la escuela: se refiere a la asunción de responsabilidad por parte de la escuela de atraer e incorporar a los padres al proceso de enseñanza aprendizaje de sus hijos: "Yo creo que el director debería incentivar e invitar a los papás a las reuniones"; "La escuela debe acercarse a conocer la realidad de sus alumnos, a través de entrevistas o de un diagnóstico"

- Relación familia - escuela iniciada por los padres: Se refiere a la asunción de responsabilidad por parte de los padres de incorporarse por iniciativa propia al proceso escolar de sus hijos: “Tenemos que comunicarnos con el profesor acerca de los niños"; "Las mamás deberían acercarse a saber cómo está su hijo en la escuela".

c) Relación familia - escuela en cuanto al ámbito de acción.

- Relación familia - escuela restringida al hogar: se entiende como tomar parte en las actividades escolares del alumno, por parte de los padres desde la casa apoyando la labor de la escuela de diferentes maneras: "Nosotras debemos preocuparnos de que el niño haga sus tareas y venga bien presentado a la escuela"; "Los padres enseñan los valores más fundamentales en el hogar y es necesario que los ensayen en esos espacios". 
- Relación familia - escuela dentro de la unidad educativa: se refiere a la presencia de los padres en la escuela para llevar a cabo distintas actividades. Participación formal: tomar parte en actividades relacionadas con lo académico ("Tenemos que venir a las reuniones lo malo es que se habla de notas nomás" ; "Debe darse a los padres la posibilidad de participar en talleres de reflexión, para que vean que lo realmente importante no es lo material, sino lo afectivo"); Participación informal: tomar parte en actividades no directamente relacionadas con lo académico ("deberían hacerse más convivencias con los papás"; "Salvo el aspecto puramente pedagógico, los padres pueden involucrarse en todo el proceso educacional").

d) Relación familia - escuela entendida como transmisión de información.

- Relación familia - escuela unidireccional: cuando la relación familia - escuela se basa en una entrega de información en una sola dirección, es decir, los padres se relacionan con la escuela sólo con la finalidad de recibir información de parte de ésta: "Solo vengo a las reuniones, pero eso de conversarle al profesor y decirle algo de mi hijo....no sé"; "Es la escuela la responsable de mantener informada a la familia, debe transmitir lo que pasa con los alumnos, la familia debe recibir lo que el profesor y el colegio le entrega y comunica".

- Relación familia - escuela bidireccional: cuando la relación familia - escuela se basa en una entrega de información recíproca, es decir, los padres se relacionan con la escuela tanto entregando información sobre sus hijos como reci- biéndola: "Con la tía tenemos una relación de amigas, ella me cuenta todos los problemas de mi hijo y vemos juntas como ayudarlo"; "Si la escuela no se nutre con esta información, si no le saca provecho a lo que viene de afuera, se verá imposibilitada de cambiar al mismo ritmo de la comunidad"..

e) Relación familia - escuela en cuanto a la jerarquía de la interacción.

- Relación familia - escuela en asimetría: cuando en la relación familia - escuela se observan distintos niveles de jerarquía, es decir, una de las partes ejerce mayor poder en la interacción, influenciando a la otra: "Cuando uno viene a hacer un reclamo del profesor al director, el director nunca le va a hallar la razón a uno"; "Los padres deben aceptar y creer en lo que nosotros hacemos como colegio".

- Relación familia - escuela en simetría: cuando en la relación familia - escuela se observa igualdad de poder e influencia al interactuar ambas partes: "Los padres deben plantear sus desacuerdos frente a la escuela, en una comunicación entre iguales"; "Nosotros debemos formar a los apoderados y ellos nos dan información".

f) Relación familia - escuela como apoyo al proceso de aprendizaje.

- Relación familia - escuela entendida como colaboración: cuando la relación que se establece entre la familia y la escuela se caracteriza por el apoyo y/o ayuda que los padres brindan a las diferentes actividades que la escuela realiza: "Nosotros podemos dar ideas a la escuela, por ejemplo, una vez en el invierno se construyó un alero para la 
lluvia"; "Los padres deben mantener buena relación con los profesores y ofrecer sus servicios para colaborar con la labor educativa".

- Relación familia - escuela entendida como co - acción: cuando la relación que se establece entre la familia y la escuela es horizontal, es decir, existe una interacción recíproca donde la familia y la escuela trabajan coordinadamente en pro del desarrollo del alumno: “Debiera haber mutua ayuda, deberían estar al tanto de los problemas de aprendizaje de sus hijos, para que juntos los ayuden más"; "Todo depende del trabajo en equipo".

g) Relación familia - escuela en cuanto a la coordinación de las partes.

- Relación familia - escuela con roles potenciados recíprocamente: cuando en la relación familia - escuela las distintas partes trabajan en forma coordinada hacia un objetivo común, donde ambas se refuerzan mutuamente: "tanto en la escuela como en la casa se debe enseñar los mismos valores, porque si nó no se saca nada"; "Hay proyectos de jardines con los papás, ellos enseñan a hacer un invernadero y también un jardín al aire libre, los papás que son expertos siguen esa parte y la profesora con sus alumnos está ocupando todo ese trabajo en sacar la parte matemática, por ejemplo, el peso de la semilla, cuánto mide la superficie, cuánto ghastamos, cuánto nos falta, es fantástico, el papá está ahí mismo y dice: esto mide más o menos un metro y medio, entonces la profesora dice:a ver, veamos, midamos, y lchiquillos ven y viven otra cosa".

- Relación familia - escuela con roles diferenciados: cuando en la relación fami- lia - escuela cada parte se desempeña en su función específica sin interferir en la acción de la otra: "En la casa se enseñan los valores y la escuela debe preocuparse de la otra parte"; "Las mamás pueden ayudar en lo que el profesor necesita y nada más, porque el profesor sabe lo que tiene que hacer".

h) Relación familia - escuela en cuanto a los beneficios que genera.

- Relación familia - escuela como generadora de beneficios instruccionales: cuando la relación familia - escuela provee de conocimientos académicos: "Se logran mayores y mejores aprendizajes de los alumnos"; "Los padres y los niños se ven beneficiados al establecer instancias de aprendizaje para ellos".

- Relación familia - escuela como generadora de beneficios afectivos: cuando la relación familia - escuela provee de instancias de desarrollo emocional: "debe existir una comunicación constante para el avance de su aprendizaje y también para reforzar la autoestima del niño"; "Esto hace que nos expresemos lo que sentimos".

- Relación familia - escuela como generadora de beneficios valóricos y formativos: cuando la relación familia - escuela provee de instancias de formación personal: "Si uno es responsable y da el ejemplo, los niños también salen responsables"; “Lograr una relación más estrecha permite el desarrollo integral del niño".

- Relación familia - escuela como generadora de beneficios sociales: cuando la relación familia - escuela provee de herramientas básicas para facilitar un desenvolvimiento adaptativo en la sociedad: "Si los niños ven que nosotras 
estamos interesadas y que participamos en las cosas de la escuela, es más fácil para ellos aprender a compartir con los demás"; "esto hace que construyamos verdaderos centros culturales".

\section{Padres participativos y no} participativos (rurales y urbanos)

En torno a la conceptualización que hacen de la relación familia - escuela los padres participativos y no participativos, se encontraron las siguientes categorías y subcategorías de respuestas, con sus respectivos porcentajes.

\begin{tabular}{|c|c|c|c|}
\hline CATEGORIA Y SUBCATEGORIA & $\begin{array}{c}\% \text { de Resp. } \\
\text { No } \\
\text { Participativos }\end{array}$ & $\begin{array}{l}\text { \% de Resp. } \\
\text { Participativos }\end{array}$ & $\begin{array}{l}\text { \% de Resp. } \\
\text { TOTAL }\end{array}$ \\
\hline I.Relación familia-escuela, de acuerdo a su naturaleza & $\mathbf{0}$ & 2.8 & 2.8 \\
\hline 1.- Como un deber & 0 & 2.8 & 2.8 \\
\hline 2.-Como un compromiso & 0 & 0 & 0 \\
\hline II.Relación familia escuela desde el punto de vista de quien la inicia & 20.0 & 30.3 & $\mathbf{5 0 . 3}$ \\
\hline 1.-Iniciada por la escuela & 3,3 & 22 & 25.3 \\
\hline 2.-Iniciada por los padres & 16.7 & 8.3 & 25 \\
\hline III.Relación familia escuela en cuanto al ámbito de acción & 26.7 & 38.9 & 65.6 \\
\hline 1.-Restringida al hogar & 0 & 2.8 & 2.8 \\
\hline 2.-Dentro de la unidad educativa & 26.7 & 36.1 & 62.8 \\
\hline 2.a)formal & 16.7 & 30.5 & 47.2 \\
\hline 2.b)informal & 10 & 5.6 & 15.6 \\
\hline $\begin{array}{l}\text { IV.Relación familia escuela entendida como transmisión de } \\
\text { información }\end{array}$ & 20 & 13.8 & 33.8 \\
\hline 1.-Unidireccional & 10 & 5.5 & 15.5 \\
\hline 2.-Bidireccional & 10 & 8.3 & 18.3 \\
\hline V. Relación familia escuela en cuanto a la jerarquía de la interacción & 6.7 & 5.5 & 13.2 \\
\hline 1.-En asimetría & 6.7 & 5.5 & 13.2 \\
\hline 2.-En simetría & 0 & 0 & 0 \\
\hline VI: Relación familia escuela como apoyo al proceso de aprendizaje & 3.3 & 11.1 & 14.4 \\
\hline 1.-Como colaboración & 3.3 & 11.1 & 14.4 \\
\hline 2.-Como coacción & 0 & 0 & 0 \\
\hline $\begin{array}{l}\text { VII. Relación familia escuela en cuanto a la coordinación de las } \\
\text { partes }\end{array}$ & $\mid 13.3$ & $\mathbf{0}$ & 13.3 \\
\hline 1.-Con roles potenciados recíprocamente & 10 & 0 & 10 \\
\hline 2.-Con roles diferenciados & 3.3 & 0 & 3.3 \\
\hline VIII. Relación familia escuela en cuanto a los beneficioa que genera & 6.6 & $\mathbf{0}$ & 6.6 \\
\hline 1.-beneficios instruccionales & 0 & 0 & 0 \\
\hline 2.-beneficios afectivos & 0 & 0 & 0 \\
\hline 3.-beneficios valóricos y formativos & 3.3 & 0 & 3.3 \\
\hline 4.-beneficios sociales & 3.3 & 0 & 3.3 \\
\hline
\end{tabular}




\section{Profesores rurales y urbanos}

En torno a la conceptualización que hacen de la relación familia - escuela los profesores rurales y urbanos, se encontraron las siguientes categorías y subcategorías de respuestas, con sus respectivos porcentajes.

\begin{tabular}{|l|l|l|l|}
\hline CATEGORIA Y SUBCATEGORIA & $\begin{array}{l}\text { \% de Resp. } \\
\text { URBAN de Resp. }\end{array}$ & $\begin{array}{l}\text { \% de Resp. } \\
\text { RURAL }\end{array}$ \\
\hline I.Relación familia-escuela, de acuerdo a su naturaleza & 3.4 & $\mathbf{8 . 7}$ & $\mathbf{1 2 . 1}$ \\
\hline 1.- Como un deber & 1.7 & 0 & 1.7 \\
\hline 2.-Como un compromiso & 1.7 & 8.7 & 10.4 \\
\hline II.Relación familia escuela desde el punto de vista de quien la inicia & $\mathbf{1 5 . 3}$ & $\mathbf{1 3}$ & $\mathbf{2 8 . 3}$ \\
\hline 1.-Iniciada por la escuela & 11.9 & 8.7 & 20.6 \\
\hline 2.-Iniciada por los padres & 3.4 & 4.3 & 7.7 \\
\hline III.Relación familia escuela en cuanto al ámbito de acción & $\mathbf{1 3 . 4}$ & $\mathbf{1 5 . 5}$ & $\mathbf{2 8 . 9}$ \\
\hline 1.-Restringida al hogar & 3.4 & 2.2 & 5.6 \\
\hline 2.-Dentro de la unidad educativa & 10 & 13.3 & 23.3 \\
\hline 2.a)formal & 5 & 8.7 & 13.7 \\
\hline 2.b)informal & 5 & 4.6 & 9.6 \\
\hline IV.Relación familia escuela entendida como transmisión de información & $\mathbf{1 0 . 2}$ & $\mathbf{6 . 5}$ & $\mathbf{1 6 . 7}$ \\
\hline 1.-Unidireccional & 3.4 & 4.3 & 7.7 \\
\hline 2.-Bidireccional & 6.8 & 2.2 & 9.0 \\
\hline V. Relación familia escuela en cuanto a la jerarquía de la interacción & $\mathbf{3 . 4}$ & $\mathbf{2 . 2}$ & $\mathbf{5 . 6}$ \\
\hline 1.-En asimetría & 3.4 & 2.2 & 5.6 \\
\hline 2.-En simetría & 0 & 0 & 0 \\
\hline VI: Relación familia escuela como apoyo al proceso de aprendizaje & $\mathbf{1 7}$ & $\mathbf{6 . 5}$ & $\mathbf{2 3 . 5}$ \\
\hline 1.-Como colaboración & 10.2 & 6.5 & 16.7 \\
\hline 2.-Como coacción & 6.8 & 0 & 6.8 \\
\hline VII. Relación familia escuela en cuanto a la coordinación de las partes & $\mathbf{1 1 . 9}$ & $\mathbf{1 7 . 4}$ & $\mathbf{2 9 . 3}$ \\
\hline 1.-Con roles potenciados recíprocamente & 8.5 & 17.4 & 25.9 \\
\hline 2.-Con roles diferenciados & 3.4 & 0 & 3.4 \\
\hline VIII. Relación familia escuela en cuanto a los beneficios que genera & $\mathbf{2 5 . 4}$ & $\mathbf{2 4 . 2}$ & $\mathbf{4 9 . 6}$ \\
\hline 1.-beneficios instruccionales & 5 & 5 & 10 \\
\hline 2.-beneficios afectivos & 6.8 & 6.8 & 13.6 \\
\hline 3.-beneficios valóricos y formativos & 10.2 & 10.2 & 20.4 \\
\hline 4.-beneficios sociales & 3.4 & 2.2 & 5.6 \\
\hline
\end{tabular}


Autoridades educacionales: Jefes de DAEM; Supervisores de DEPROES y; Directores de Establecimientos educacionales
En torno a la conceptualización que hacen de la relación familia - escuela las autoridades educacionales, se encontraron las siguientes categorías y subcategorías de respuestas, con sus respectivos porcentajes.

\begin{tabular}{|l|l|l|l|}
\hline CATEGORIA Y SUBCATEGORIA & $\begin{array}{l}\text { \%deresp } \\
\text { SUPERV }\end{array}$ & $\begin{array}{l}\text { \%desp resp } \\
\text { DAEM }\end{array}$ & $\begin{array}{l}\text { resp } \\
\text { DIRECT }\end{array}$ \\
\hline I.Relación familia-escuela, de acuerdo a su naturaleza & $\mathbf{7}$ & $\mathbf{0}$ & $\mathbf{7 . 5}$ \\
\hline 1.- Como un deber & 4.7 & 0 & 6.1 \\
\hline 2.-Como un compromiso & 2.3 & 0 & 1.4 \\
\hline II.Relación familia escuela desde el punto de vista de quien la inicia & $\mathbf{1 6 . 3}$ & $\mathbf{2 8 . 6}$ & $\mathbf{1 6}$ \\
\hline 1.-Iniciada por la escuela & 14 & 22.9 & 13 \\
\hline 2.-Iniciada por los padres & 2.3 & 5.7 & 3.0 \\
\hline III.Relación familia escuela en cuanto al ámbito de acción & $\mathbf{1 8 . 6}$ & $\mathbf{1 1 . 4}$ & $\mathbf{2 7 . 4}$ \\
\hline 1.-Restringida al hogar & 0 & 0 & 1.4 \\
\hline 2.-Dentro de la unidad educativa & 18.6 & 11.4 & 26 \\
\hline 2.a)formal & 11.6 & 8.5 & 16 \\
\hline 2.b)informal & 7 & 2.9 & 10 \\
\hline IV.Relación familia escuela entendida como transmisión de información & $\mathbf{7 . 0}$ & 5.7 & $\mathbf{1 . 4}$ \\
\hline 1.-Unidireccional & 4.7 & 0 & 1.4 \\
\hline 2.-Bidireccional & 2.3 & 5.7 & 0 \\
\hline V. Relación familia escuela en cuanto a la jerarquía de la interacción & $\mathbf{2 . 3}$ & $\mathbf{5 . 7}$ & $\mathbf{5 . 7}$ \\
\hline 1.-En asimetría & 2.3 & 5.7 & 4.3 \\
\hline 2.-En simetría & 0 & 0 & 1.4 \\
\hline VI: Relación familia escuela como apoyo al proceso de aprendizaje & 9.3 & $\mathbf{2 0 . 9}$ & $\mathbf{8 . 5}$ \\
\hline 1.-Como colaboración & 2.3 & 15.2 & 7.2 \\
\hline 2.-Como coacción & 7 & 5.7 & 1.3 \\
\hline VII. Relación familia escuela en cuanto a la coordinación de las partes & $\mathbf{0}$ & $\mathbf{1 6 . 3}$ & $\mathbf{0}$ \\
\hline 1.-Con roles potenciados recíprocamente & 0 & 14 & 0 \\
\hline 2.-Con roles diferenciados & 0 & 23 & 0 \\
\hline VIII. Relación familia escuela en cuanto a los beneficios que genera & $\mathbf{2 3 . 2}$ & $\mathbf{2 3 . 8}$ & $\mathbf{2 3 . 2}$ \\
\hline 1.-beneficios instruccionales & 4.7 & 12.4 & 10.1 \\
\hline 2.-beneficios afectivos & 2.3 & 5.7 & 10.1 \\
\hline 3.-beneficios valóricos y formativos & 7 & 0 & 3 \\
\hline 4.-beneficios sociales & 9.2 & 5.7 & 0 \\
\hline
\end{tabular}

\section{Discusión}

\section{A nivel de padres}

Los padres participativos tienden a generar más opiniones indicadoras de que es la unidad educativa la que debiera iniciar la relación familia - escuela, mientras que los padres no participativos tienden a atribuir más esa responsabilidad a los padres. Esto podría explicarse porque los padres que participan consideran que los profesores tienen la autoridad legítima y que deberían hacer uso de ella para generar el acercamiento de los padres; los que no participan tienden a ver a la escuela como autoritaria y la obligatoriedad de sus disposiciones como una condición no deseada, ante la cual deciden restarse de participar.

Los padres participativos tienden a circunscribir el ámbito de acción de la familia tanto al ambiente hogareño como al escolar y en este caso lo restringen a aspectos formales, quizá influidos por la labor formal que tradicionalmente desempeñan. Los padres no participativos restringen la acción al am- 
biente escolar, tanto en lo formal como en lo informal. Esto podría estar indicando que quienes no participan en las directivas de los microcentros tienden a buscar otras instancias de participación, además de las consideradas tradicionalmente como formales.

Los participativos tienden a asignar mayor valor a la bidireccionalidad, fenómeno atribuible a su rol en la unidad educativa, caracterizado por una mayor conexión con los docentes, mayor habilidad en la negociación y toma de decisiones, además de la propia autoridad y legitimidad social que deriva de sus cargos. Los no participativos asignan igual porcentaje a la bidireccionalidad $\mathrm{y}$ unidireccionalidad, por lo que parecieran no tener una postura tan clara al respecto.

Respecto a la jerarquía relacional ambos grupos de padres tienden a percibir la relación familia - escuela como asimétrica, donde la unidad educativa ejerce mayor poder e influencia sobre la familia. Lo anterior se entendería al observar una realidad social y educacional chilena que aún no ha interiorizado el valor de los aportes que todos los individuos pueden hacer, sumado a la noción de autoridad que detenta el rol docente, especialmente en el sector rural, que dificultaría una relación más horizontal.

Todos, fundamentalmente los participativos, consideran que el apoyo de la familia al proceso de enseñanza aprendizaje es de colaboración. Esto podría explicarse por una tendencia a considerar el quehacer de la familia restringido a una labor de apoyo puramente tradicional. El que no consideren la posibilidad de una coacción podría deberse a que la realidad sociocultural no incentiva el trabajo en equipo, lo que impide que los padres consideren la coacción como una posibilidad de aunar esfuerzos con la unidad educativa y co - participar del proceso.

Solo los padres no participativos se pronuncian en la categoría sobre la coordina- ción de las partes y le asignan una mayor importancia a la potenciación de los roles entre familia y escuela. Esta característica puede evidenciar una probable necesidad sentida de ser valorados y considerados capaces de aportar al proceso educativo.

Los participativos no se expresan en cuanto a los beneficios que genera la relación familia escuela. Una posible explicación sería que para ellos, el solo hecho de participar y desempeñar un rol protagónico en el ámbito educativo les proporcionaría beneficios considerados como naturales. En un bajo porcentaje, los no participativos señalan beneficios de esta relación, lo que podría explicarse probablemente, por una percepción de algunas carencias con respecto a la estructura educacional.

De la comparación de las opiniones de los padres con el concepto de participación formulado teóricamente, es posible concluir una correspondencia en los factores considerados para la conceptualización del proceso, pero las apreciaciones que los padres tienen son más abarcadoras y más comprensivas del proceso. Los padres consideran, a diferencia del concepto teórico, quién inicia la conexión familia escuela; el aspecto comunicacional de la relación; la diferencia jerárquica que prima y los beneficios potenciales de la relación.

Los padres no participativos plantean más categorías para conceptualizar la relación familia escuela, lo que sería acorde a una probable habituación de los que participan con respecto a la participación y sus beneficios y, con la sensibilidad que produciría el no gozar de las condiciones para la participación, lo que generaría una tendencia a ampliar la visión de lo que es importante en una relación entre los sistemas.

\section{A nivel de profesores}

Los profesores rurales opinan en mayor porcentaje que la relación familia escuela 
debe ser de compromiso. Al respecto, se puede argumentar que en este contexto, la cercanía entre las personas es mayor, ya que generalmente hay menos gente $y$, porque al haber, probablemente, una sola escuela en el sector, se la ve como la opción que tienen los niños de salir adelante y llegar a ser más de lo que han sido los padres. Posiblemente se valoraría el rol que ejerce el profesor y la escuela y la importancia del compromiso de la familia y la escuela para la formación del niño.

Un mayor número de opiniones de profesores urbanos y rurales indican que la relación familia - escuela debiera ser iniciada por la unida educativa. Esto indicaría que los profesores habrían asumido la responsabilidad de lo que ocurre actualmente en las escuelas, en las cuales ellas son las encargadas de atraer a la familia, pero que también implica que aún no se habría tomado plena conciencia de que esta relación podría ser iniciada por ambos sistemas.

Con respecto al ámbito de acción de la relación, se encontró que los profesores rurales tienden a circunscribirla especialmente al ámbito escolar y dan mayor importancia a lo formal que los profesores urbanos. Esto se debería, en alguna medida, porque la percepción de la participación de los padres en las escuelas rurales se da en el ámbito formal y se considera poco importante para el proceso de enseñanza aprendizaje su participación en otros ámbitos. Los profesores esperarían la respuesta de los padres hacia acciones determinadas por la escuela y no valorarían otros aportes que pudieran hacer, probablemente, debido a su bajo nivel instruccional.

Los profesores rurales tienden a asignar mayor valor a la unidireccionalidad en la transmisión de información. Lo anterior podría indicar que los profesores en estos sectores tenderían a considerar poco relevante el aporte de la gente, probablemente, por su bajo nivel instruccional. Los profesores de las escuelas urbanas tienden a opinar que tanto la familia como la escuela deberían compartir la información para que el proceso de enseñanza aprendizaje sea más provechoso.

Profesores urbanos y rurales tiende a percibir la relación familia escuela como asimétrica, con un mayor poder e influencia de la unidad educativa sobre la familia. . Esta opinión evidencia la influencia de una realidad social y educacional chilena que aún no ha interiorizado el valor de los aportes que todos los individuos pueden hacer, el valor de la participación, sumado a la noción de autoridad que detenta el rol docente.

Respecto a la coordinación de las partes, profesores urbanos y rurales valoran la potenciación de los roles. Esta idea se apoya en una noción compartida de que la familia y la escuela deben ir hacia un objetivo común, la educación del niño; especialmente en la parte afectiva y valórica la que, con la reforma educacional, ya no es considerada función sólo de la familia, sino que, con los objetivos transversales, se hace compartida con la escuela.

Profesores rurales y urbanos asignan gran importancia a los beneficios que genera la relación familia escuela, especialmente los afectivos y valóricos. Esto, además de apoyarse en aspectos de formación, podría haberse potenciado por una posible influencia de la reforma educacional en ellos, la cual recalca el valor de estos aspectos para la formación integral del niño, principalmente bajo la forma de la formulación de objetivos transversales y de la clarificación del proyecto educativo de cada establecimiento.

De las opiniones de los docentes se puede concluir que su visión de participación considera más factores que la definición teórica del concepto. Toman en cuenta quién inicia la relación, los beneficios que genera y el flu- 
jo de comunicación que se debe dar para mantenerla. La visión en ambos sectores considera la participación como apoyo a la labor docente, con una jerarquía asimétrica en la relación, donde el profesor la inicia, comunica y determina que es lo que se requiere de la familia para el proceso de enseñanza aprendizaje. Ambos grupos de profesores dan importancia al trabajo conjunto que debe realizar la familia con la escuela para beneficiar la formación integral de los educandos. Para los profesores urbanos la comunicación establecida en la relación familia escuela debe ser bidireccional y los profesores rurales privilegian una comunicación unidireccional y una participación fundamentalmente formal de los padres.

\section{A nivel de autoridades educacionales}

Directores, jefes de DAEM y supervisores, señalan que la relación familia escuela debe ser iniciada por ésta, lo que podría explicarse por una mayor interiorización de la política de "puertas abiertas" que promueve la reforma educacional. Todas las autoridades, especialmente los supervisores, opinan que la acción de la familia debe ampliarse del hogar al ámbito escolar, pero circunscrita a instancias formales. Esto podría indicar cierto nivel de asociación entre la tendencia a implicarse con el movimiento que promueve la reforma y la jerarquía que detenta cada persona dentro del sistema educacional.

Respecto a la coordinación de las partes los supervisores plantean en mayor medida, una potenciación de los roles producto de una adecuada relación familia escuela. Esto podría deberse a su mejor manejo teórico de los beneficios que proporciona este tipo de conexión, una mayor identificación con las políticas ministeriales de promoción de la participación y democratización de las relaciones sociales. Que los directores planteen en menor grado esta potenciación de roles se podría deber a una falta de disposición a compartir la autoridad en cuanto a la toma de decisiones, heredada de la forma tradicional de conducir la educación, previa a la promoción de la participación como estrategia educacional.

Todas las autoridades plantean que a partir de la relación familia escuela se generarían beneficios, principalmente, instruccionales. Lo anterior se entiende por la primacía de la orientación al logro académico en el sistema y por la necesidad de generar mejoras en este plano, dadas las evaluaciones a las que se ven sometidos y a los beneficios que esto les asegura.

Existe una alta coincidencia entre el concepto teórico de participación de los padres en el proceso de enseñanza aprendizaje de los hijos y lo planteado por las autoridades, tanto en la amplitud de los ámbitos que incluye como en la no-especificidad de los aspectos que considera. Esto podría explicar la tendencia a no explotar nuevos puntos de conexión y de relación entre la familia y la escuela y la tendencia de las autoridades a manejarse en el plano puramente conceptual de lo que es la participación, sin dar ejemplos ni instancias concretas para fortalecer esta relación ni de los beneficios extra - académicos que podría generar. Las diferencias entre los tres estamentos podrían explicarse por la visión más macro que se tendría del proceso de enseñanza aprendizaje a medida que se asciende en la escala jerárquica. Los supervisores se muestran motivados por políticas nacionales acerca de la democratización de las relaciones sociales; los jefes de DAEM estarían motivados por mejorar la realidad educacional de una comuna completa y, los directores se muestran más motivados en la realidad particular de sus escuelas, en sus experiencias personales acerca de la relación familia escuela. 


\section{Conclusiones}

De acuerdo a la investigación realizada, es pertinente destacar que la definición teórica de Participación de los padres en el proceso de enseñanza aprendizaje de los hijos no es absolutamente coincidente con lo planteado por los actores directos del proceso educativo, difieren tanto en la gama de aspectos considerados en la definición como en la profundidad de análisis. En este sentido, es posible plantear que la participación no es un concepto absoluto, sino un continuo dentro del cual se pueden verificar diferentes grados en cuanto al nivel de involucramiento y acercamiento de los padres con la escuela. Además, es en el contexto de aprendizaje donde la participación de padres toma su real relevancia, sin importar la definición nominal que se haga desde otras instancias, sino la idea -en cualquier nivel de amplitud o certeza- que mantengan los actores de ella y que les hace actuar de tal o cual manera.

Los padres se muestran conscientes de los beneficios que podrían generarse a partir de una mayor y más eficaz relación entre familia y escuela. Entre los padres designados como "no-participativos", esta tendencia se inclina más hacia factores de carácter no directamente instruccionales y a las instancias informales o novedosas de interrelación, evidenciando la necesidad de las instituciones de considerar acoger a estos apoderados, como un punto inicial en el cumplimiento de objetivos más relacionados con la formación de sus hijos.

De parte de las autoridades se aprecia una tendencia a implementar la participación desde un plano eminentemente teórico, sin profundizar o pensar en lo que la gente realmente está entendiendo y valorando, por lo tanto, sin llevar aún un registro más detallado de cómo se está a llevando a cabo en las escuelas y con qué niveles de participación.
Las diferencias establecidas cuando se relacionan la familia y la escuela, la visión tradicionalista para concebir la participación, el temor de los profesores a perder la exclusividad en el tema educacional y la no-consideración de todos los actores del proceso educativo en la ideación e implementación de las medidas de la Reforma, ha impedido que la participación sea entendida como un proceso capaz de generar beneficios significativos tanto para la realidad educacional como social más amplia. Es importante considerar que todos estos son aspectos perfectamente modificables en un contexto adecuado de relación y comunicación, después de haber hecho un análisis realista de las metas y posibilidades de cada institución dispuesta a superarlos.

De lo anterior es posible señalar que la participación de los padres en el proceso de enseñanza - aprendizaje de los hijos es una especie de continuo, que varía en grados de involucramiento entre los dos sistemas y en donde el ideal perseguido dependerá de la naturaleza particular de la unidad educativa, de la singularidad del núcleo familiar, de las condiciones de la comunidad en la cual está inserto y por la compleja realidad que se construye a partir de la interrelación de estos sistemas.

Con estos antecedentes es aconsejable y necesario hacer partícipes a todos los agentes que intervienen en el sistema educacional, tanto en la creación como en la puesta en marcha de los cambios en el sistema. Este es un requisito básico para aumentar la motivación y compromiso de sus actores para lograr con éxito las metas propuestas en conjunto.

Finalmente se considera interesante seguir investigando en este tema para avanzar en el proceso de democratización de la sociedad y favorecer los cambios que trae consigo la Reforma Educacional Chilena. Al res- 
pecto, faltan datos acerca de cómo se vive este concepto en la enseñanza media, en donde recién comienzan a introducirse los cambios de modernización.

\section{Referencias}

Arancibia, V. (1997). Efectividad Escolar: Un análisis comparado. Santiago: Estudios Públicos.

Assaél, J., Cerda, A. y Rodas, M. (1996). Una mirada evaluativa a los proyectos de mejoramiento educativo (PME). Revista de Educación, 236, 51-55.

CANTÓN, I. (1996). Manual de organización de centros educativos. Barcelona: Oikos-Tau.

FERNÁNDEZ, G. y GUERRERO, S. (1996) Espacios de participación en la escuela, desde la reflexión a la acción. En: Documento no 10, CIDE, Santiago.

Ministerio de EdUCACIÓN (1995). La Reforma en Marcha. Santiago: Ministerio de Educación.

Ministerio de Educación (1998). La Reforma en Marcha: Buena educación para todos. Santiago: Ministerio de Educación.

NeIRA, D. (1994). Introducción a temas fundamentales de educación. Concepción: Universidad de Concepción, Proyecto Desarrollo de la Docencia.

Pardo, S., Rodríguez, C., Rodríguez, P., Sáez, X., Salvatierra, T. Y Venegas, M. (1998). Participación de los padres y/o apoderados en el proceso de enseñanza aprendizaje: concepto, dificultades y facilitadores. Seminario para optar al grado de Licenciado en Psicología, Universidad de Concepción, Concepción.

Revista de Educación (1998). Familia y escuela: Una alianza necesaria. Revista de Educación, № 253, pp. 31 - 38.

\section{Apéndice}

\section{Pauta para entrevista y discusión grupal}

1. ¿Cómo cree usted que debiera ser la relación entre la familia y la escuela o entre la escuela y la familia?.

2. ¿Por qué considera importante que la relación se dé de esta forma?.

3. ¿Quiénes cree usted que son los responsables de que la relación se dé de esta forma?

4. ¿Cuál debiera ser el rol de los profesores en el establecimiento de la relación familia- escuela; escuela - familia?

5. ¿Cuál debiera ser el rol de los padres en el establecimiento de la relación familia- escuela; escuela - familia?

6. ¿Cuál debiera ser el rol de las autoridades en el establecimiento de la relación familia- escuela; escuela - familia?

7. ¿Qué condiciones cree usted que favorecen el que se dé una relación de este tipo?

8. ¿Qué condiciones cree usted que dificultan el que se dé una relación de este tipo?

9. ¿Cómo cree usted que se da la relación familia - escuela o escuela - familia en la actualidad? ¿Qué mejoraría y qué dejaría igual?. 